\title{
Safety and Tolerability of the Adeno-Associated Virus Vector, AAV6.2FF, Expressing a Monoclonal Antibody in Murine and Ovine Animal Models
}

\author{
Amira D. Rghei ${ }^{1,+}$, Laura P. van Lieshout ${ }^{2,+}$, Benjamin M. McLeod ${ }^{1}$, Yanlong Pei ${ }^{1}$, Jordyn A. Lopes ${ }^{1}$, \\ Nicole Zielinska ${ }^{1}$, Enzo M. Baracuhy ${ }^{1}$, Brenna A. Y. Stevens ${ }^{1}$, Sylvia P. Thomas ${ }^{1}$, Jacob G. E. Yates ${ }^{1}$, \\ Bryce M. Warner ${ }^{3}$ (D), Darwyn Kobasa ${ }^{3}{ }^{\mathbb{D}}$, Hugues Fausther-Bovendo ${ }^{4}$, Gary P. Kobinger ${ }^{4}$, Khalil Karimi ${ }^{1}$, \\ Brad Thompson $^{2}$, Byram W. Bridle ${ }^{1}$ (D) , Leonardo Susta ${ }^{1}$ (D) and Sarah K. Wootton ${ }^{1, *(D)}$
}

check for updates

Citation: Rghei, A.D.; van Lieshout, L.P.; McLeod, B.M.; Pei, Y.; Lopes, J.A.; Zielinska, N.; Baracuhy, E.M.; Stevens, B.A.Y.; Thomas, S.P.; Yates, J.G.E.; et al. Safety and Tolerability of the Adeno-Associated Virus Vector, AAV6.2FF, Expressing a Monoclonal Antibody in Murine and Ovine Animal Models. Biomedicines 2021, 9, 1186. https://doi.org/10.3390/ biomedicines 9091186

Academic Editor:

Kenneth Lundstrom

Received: 16 August 2021

Accepted: 7 September 2021

Published: 9 September 2021

Publisher's Note: MDPI stays neutral with regard to jurisdictional claims in published maps and institutional affiliations.

Copyright: (c) 2021 by the authors. Licensee MDPI, Basel, Switzerland. This article is an open access article distributed under the terms and conditions of the Creative Commons Attribution (CC BY) license (https:/ / creativecommons.org/licenses/by/ $4.0 /)$.
1 Department of Pathobiology, Ontario Veterinary College, University of Guelph, Guelph, ON N1G 2W1, Canada; arghei@uoguelph.ca (A.D.R.); bmcleo03@uoguelph.ca (B.M.M.); ypei@ovc.uoguelph.ca (Y.P.); jlopes@uoguelph.ca (J.A.L.); zielinsn@uoguelph.ca (N.Z.); ebaracuh@uoguelph.ca (E.M.B.); bsteve04@uoguelph.ca (B.A.Y.S.); sthoma13@uoguelph.ca (S.P.T.); jyates01@uoguelph.ca (J.G.E.Y.); kkarimi@uoguelph.ca (K.K.); bbridle@uoguelph.ca (B.W.B.); lsusta@uoguelph.ca (L.S.)

2 Avamab Pharma Inc., 120, 4838 Richard Road SW, Calgary, AB T3E 6L1, Canada; lauravan93@hotmail.com (L.P.v.L.); bt@kickshawventures.com (B.T.)

3 Zoonotic Diseases and Special Pathogens, Public Health Agency of Canada, Winnipeg, MB R3E 3R2, Canada; bryce.warner@canada.ca (B.M.W.); darwyn.kobasa@canada.ca (D.K.)

4 Département de Microbiologie-Infectiologie et D'immunologie, Université Laval, Quebec City, QC G1V 0A6, Canada; faustlbv@gmail.com (H.F.-B.); gary.kobinger@crchudequebec.ulaval.ca (G.P.K.)

* Correspondence: kwootton@uoguelph.ca; Tel.: +1-519-824-4120 (ext. 54729)

+ These authors contributed equally.

Abstract: Adeno-associated virus (AAV) vector mediated expression of therapeutic monoclonal antibodies is an alternative strategy to traditional vaccination to generate immunity in immunosuppressed or immunosenescent individuals. In this study, we vectorized a human monoclonal antibody (31C2) directed against the spike protein of SARS-CoV-2 and determined the safety profile of this AAV vector in mice and sheep as a large animal model. In both studies, plasma biochemical parameters and hematology were comparable to untreated controls. Except for mild myositis at the site of injection, none of the major organs revealed any signs of toxicity. AAV-mediated human IgG expression increased steadily throughout the 28-day study in sheep, resulting in peak concentrations of $21.4-46.7 \mu \mathrm{g} / \mathrm{mL}$, demonstrating practical scale up from rodent to large animal models. This alternative approach to immunity is worth further exploration after this demonstration of safety, tolerability, and scalability in a large animal model.

Keywords: adeno-associated virus (AAV) vector; vectored immunoprophylaxis; monoclonal antibody; safety; tolerability; large animal model

\section{Introduction}

Monoclonal antibody $(\mathrm{mAb})$ therapies have become a popular strategy for combatting infectious diseases, especially those without licensed vaccines or effective therapeutic treatment [1-3]. Recently, there has been an increase in research into mAbs that provide protection against a wide variety of viruses and bacteria, including SARS-CoV-2 [1,4-7]. Though mAbs are effective as therapeutics, production of clinical grade mAbs for passive immunization is costly and the resulting immunity is short-lived [8]. An alternative approach to passive immunization is vectorized antibody expression, in which an adenoassociated virus (AAV) vector is used to transfer a mAb gene to host cells that subsequently produce and secrete antibody into the blood $[9,10]$. In this manner, antibody expression 
is continuously manufactured within treated patients, providing immunity for much extended periods compared to passive antibody transfer.Vectorized antibody expression has been highly effective at protecting animal models from various infectious diseases including HIV, Influenza virus, Ebola virus and Clostridium difficile [11-14].

$\mathrm{AAV}$ vectors provide sustained expression of therapeutic transgenes; however, the potential of the AAV capsid or transgene product resulting in toxicity is a potential safety concern. To the best of our knowledge, there are no published studies examining the toxicity of AAV vectorized antibody expression; however, there is an abundance of preclinical and clinical studies that underscore the safety and tolerability of AAV-based therapies $[15,16]$. In this study, we selected a triple mutant AAV6 capsid, AAV6.2FF, as our vector of choice, due to its superior ability to transduce both muscle and lung tissue, as shown in murine and hamster animal models $[13,14,17]$. Here, we use AAV6.2FF to evaluate the safety and tolerability of this approach through hematology, blood biochemistry and histopathology and the feasibility of vectorized antibody expression in murine and ovine animal models to reach potentially therapeutic plasma antibody concentrations. Sheep represent a large, outbred animal model that is more genetically diverse than mice, and thus may be more reflective of what could happen in humans administered AAV vectors expressing mAbs. We hypothesized that AAV6.2FF-vectorized expression of human mAb, 31C2 would lead to robust antibody expression and would not negatively impact the immune system and general health of both murine and ovine animal models when intramuscularly administered.

\section{Materials and Methods}

\subsection{AAV Vector Production}

The heavy and light chain variable region gene sequences for monoclonal antibody 31C2 (kindly provided by H Fausther-Bovendo and G Kobinger, Laval University) were expressed from a single gene expression cassette under the control of a CASI promoter to generate human IgG heavy chain and lambda light chain polypeptides separated by a furinF2A self-cleaving peptide [18]. A woodchuck hepatitis virus post transcriptional regulatory element (WPRE) and SV40 polyadenylation signal were included in the vector and the entire expression cassette was flanked by AAV2 inverted terminal repeats to facilitate packaging. AAV production was carried out as previously described using adherent HEK293 cells and heparin affinity chromatography [19].

\subsection{AAV Titration}

$A A V$ vector genome titers were determined by qPCR. Vector samples were pre-treated sequentially with DNase (Promega M6101, Madison, WI, USA) and proteinase K (Invitrogen LSAM2546 Carlsbad, CA, USA) and purified by Qiagen Blood and Tissue Kit (Qiagen 69504, Germantown, Maryland). DNA samples were analyzed by qPCR using a TaqMan primer and probe set against the human $\operatorname{IgG}$ heavy chain sequence: forward primer 5'-TGCAACGTGAATCACAAGC-3', reverse primer 5'-GCATGTGTGAGTTTTGTCAC-3' and probe $5^{\prime}$ FAM/CACCAAGGT/Zen/GGACAAGAAA GTTGAGCCC/3IABkFQ (Integrated DNA Technologies Coralville, IA, USA), Luna universal qPCR master mix (New England Biolabs M3003, Ipswich, MA, USA) and a LightCycler 480 (Roche Nutly, NJ, USA) thermal cycler.

\subsection{Animal Experiments}

All animal experiments were approved by the University of Guelph Animal Care Committee in accordance with Canadian Council on Animal Care (CCAC) guidelines. Five-week-old BALB/c mice were purchased from Jackson Labs (Bar Harbor, ME, USA) and allowed to acclimatize for one week prior to study commencement. All mouse groups contained equal numbers of male and female animals. Mice received an intramuscular injection of AAV vector diluted in $1 \times$ phosphate buffered saline (PBS) $\mathrm{pH} 7.4$ to a final volume of $40 \mu \mathrm{L}$, which was administered to the gastrocnemius muscle using a tuberculin syringe. In-life blood draws were completed by saphenous bleed using EDTA microvettes (Sarstedt 
Inc, Newton, NC, USA). At endpoint, mice were deeply anesthetized with isoflurane for terminal retro-orbital blood collection in non-heparinized micro-hematocrit capillary tubes (DWK Life Sciences, Rockwood, TN, USA) or euthanized by isoflurane overdose for BAL. $800 \mu \mathrm{L}$ of PBS was administered to lungs using a shielded 20G catheter (BD, Franklin Lakes, NJ, USA) and withdrawn after $2 \mathrm{~min}$. The BALF was centrifuged at $2000 \mathrm{rpm}$ for two minutes to pellet debris and the supernatant was collected.

Newborn male Dorset lambs nursed colostrum, as well as supplemental bovine dried colostrum, from their mothers for 48 hours prior to being adapted to consume milk replacer from a bottle feeder for the remainder of this study. Lambs were administered AAV6.2FF-31C2 by weight at a dose of $5 \times 10^{12} \mathrm{VG} / \mathrm{kg}$. Vector was diluted in $1 \mathrm{X}$ PBS to a volume of $1 \mathrm{~mL}$ and multiple injections were delivered to the rump muscle, separated by approximately three inches. Injection sites were marked with spray paint to enable tissue collection from the site of injection at necropsy. Weekly blood draws were completed by jugular bleed using the Vacutainer blood collection system (BD, Franklin Lakes, NJ, USA).

Multiple sets of $25 \mathrm{mg}$ samples of sheep lung tissue were rinsed with PBS and collected in $1.5 \mathrm{~mL}$ flat bottom Sarstedt O-ring tube (Sarstedt Inc, Newton, NC, USA). $500 \mu \mathrm{L}$ RIPA buffer containing protease inhibitor (Thermo Fisher, Waltham, MA, USA) was added to the tube along with one $1 / 4^{\prime \prime}$ ceramic bead (MP 6540424, Santa Ana, CA, USA) and homogenized on the Precellys 24 tissue homogenizer (Thermo Fisher, Waltham, MA, USA) at $6000 \mathrm{rpm}$ for $2 \times$ one-minute cycles. Homogenized samples were then centrifuged at $13,000 \mathrm{rpm}$ for $20 \mathrm{~min}$ at $4{ }^{\circ} \mathrm{C}$ to pellet cell debris. Supernatant was collected for subsequent hIgG quantification.

\subsection{Enzyme-Linked Immunosorbent Assays (ELISAs)}

Commercially available ELISA kits for human IgG (Abcam 195215, Cambridge, MA, USA) murine IgG (Abcam 157719, Cambridge, MA, USA) and ovine IgG (Abcam 190546, Cambridge, MA, USA) were used to determine plasma immunoglobulin concentrations. Human IgG concentrations in lavage fluid was expressed as human IgG concentration per $\mathrm{mL}$ of lavage sample.

Anti-AAV6.2FF capsid antibodies in sheep serum were detected using half well 96 well plates (Corning, NY, USA) coated with $30 \mu \mathrm{L}$ of $1 \times 10^{10} \mathrm{VG} / \mathrm{mL}$ AAV6.2FF-Luciferase overnight at $4{ }^{\circ} \mathrm{C}$. Plates were then washed three times using $0.2 \%$ PBS-Tween20 (PBST) and blocked using SuperBlock buffer (Fisher 37515, Waltham, MA, USA) for $30 \mathrm{~min}$. Two-fold plasma dilutions beginning at 1:400 were incubated at $37^{\circ} \mathrm{C}$ for $60 \mathrm{~min}$ and then washed three times with $0.1 \%$ PBS-T. Donkey anti-sheep HRP conjugated secondary antibody (Thermo Fisher, Waltham, MA, USA) was diluted to 1:5000, added to each well and incubated at $37^{\circ} \mathrm{C}$ for $60 \mathrm{~min}$. The plate was then washed three times with $0.2 \%$ PBS-T and incubated with TMB substrate (Pierce PI34021 Waltham, MA, USA) for $15 \mathrm{~min}$ at room temperature. Reciprocal titer was defined as the highest serial serum dilution that gave an OD650 value 2-fold greater than the mean of the negative control wells.

\subsection{Hematology and Clinical Chemistry}

Terminal murine EDTA whole blood samples were submitted to the Animal Health $\mathrm{Lab}$ (AHL) at the University of Guelph (Guelph, ON, Canada) for manual leukocyte differential and a custom clinical chemistry panel using a Cobas 6000. Ovine EDTA and heparin whole blood samples were submitted weekly to the AHL for comprehensive complete blood count using an AVIDA 2120 and custom clinical chemistry panel using a Cobas 6000. Ovine reference ranges from the University of Guelph Animal Health Lab and University California Davis Veterinary Medical Teaching Hospital were consulted for analysis.

\subsection{Cytokine Analysis}

Murine plasma samples from the day 7 and day 28 cohorts were submitted to EveTechnologies (Calgary, AB, Canada) for mouse cytokine analysis using the Luminex 200 system 
(Luminex, Austin, TX, USA). Ten cytokines were simultaneously measured in the samples using Eve Technologies' Mouse Focused 10-Plex Discovery Assay®(MilliporeSigma, Burlington, MA, USA) according to the manufacturer's protocol. Plasma samples were diluted 2-fold with PBS pH 7.5 prior to analysis. Assay sensitivities of these markers range from $0.4-10.9 \mathrm{pg} / \mathrm{mL}$ for the 10-plex.

\subsection{Histology and Immunohistochemistry}

Tissue samples were fixed for 24 hours in 10\% formalin, washed twice with PBS and stored in $70 \%$ ethanol. Fixed tissues were submitted to AHL for histology processing and H\&E staining. Pathology interpretation was completed blinded by a board-certified anatomic pathologist. Additional slides were submitted to AHL for IHC staining using an antibody against human IgG (Abcam 181236, Cambridge, MA, USA) at a 1:100 dilution following antigen retrieval using citrate buffer $\mathrm{pH} 6$ and blocking with Protein Block Serum-Free (DAKO Corporation, Hamburg, Germany). EnVision+ system- HRP anti-Rabbit labelled polymer was used as secondary antibody according to the manufacturer's instruction. NovaRed was used for HRP detection and slides were counterstained using hematoxylin. IHC staining was automated using Dako Autostiner Plus (DAKO Corporation, Hamburg, Germany).

\subsection{AAV Vector Biodistribution}

Tissues samples were frozen at necropsy and DNA was extracted from $25 \mathrm{mg}$ of tissue using a Blood and Tissue Kit (Qiagen 69504 Germantown, MD, USA) and vector genome copy number was determined by qPCR as described above. DNA concentration was determined using Nanodrop and the vector genome copy number was normalized to the total genomic DNA concentration.

\subsection{Statistics}

Graphpad Prism 9 software (San Diego, CA, USA) was used to generate all statistical analyses. Mean and standard deviation are displayed on all graphs with the exception of the data from the lamb study in which each animal is plotted individually. One-way analysis of variance was used to evaluate the differences within cohorts. Tukey's multiple comparison test was used as a post-test. An unpaired One-way ANOVA was used to compare the mouse weights over time. $p$ values of $<0.05$ was considered significant.

\section{Results}

\subsection{Confirmation of AAV6.2FF-31C2 Vector Expression}

Heavy and light chain variable region sequences for $31 \mathrm{C} 2$ were kindly provided by collaborators working to isolate and characterize mAbs from COVID-19 patient samples. The AAV vector genome expressing 31C2 as human IgG1 under the control of a strong ubiquitous promoter was packaged using the AAV6.2FF capsid. Groups of male and female $(n=4) \mathrm{BALB} / \mathrm{c}$ mice were injected intramuscularly (IM) with $2 \times 10^{10}$ vector genomes (VG) of AAV6.2FF-31C2. Blood draws occurred on day 0, 7, 21, 28, 42,70 and 98 post AAV administration and plasma was analyzed for human IgG (hIgG) concentration to evaluate transgene expression (Figure 1A). 
At approximately 14 weeks post vector administration, these mice were sacrificed, and bronchoalveolar lavages (BAL) were performed and hIgG in the lavage fluid was quantified (Figure 1B). BAL fluid (BALF) trended similarly to plasma hIgG concentrations; expressing 31C2 had an average of 121.6 nanograms (ng)/mL lavage fluid, demonstrating robust plasma concentrations are required for antibody diffusion into the lung mucosal surface. Differences in the hIgG levels in both the plasma and BALF varied based on sex differences and due to variability during IM administration of the vector and efficiency of BALF collection.

A

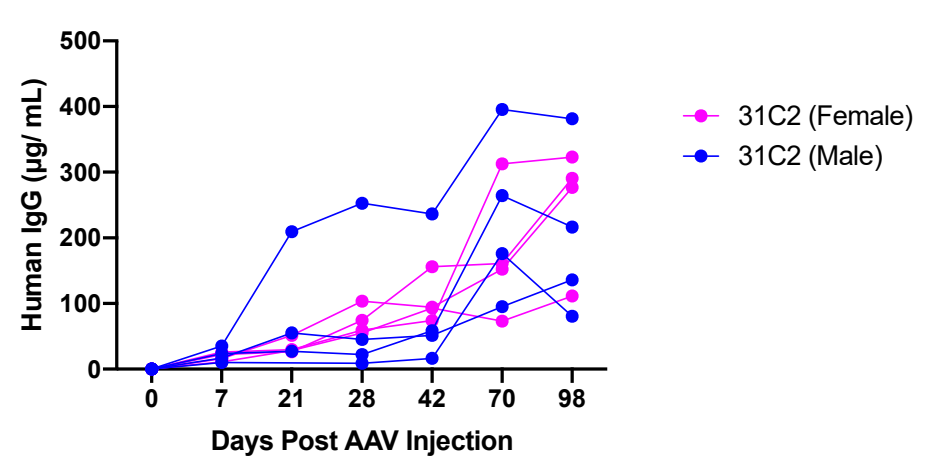

B

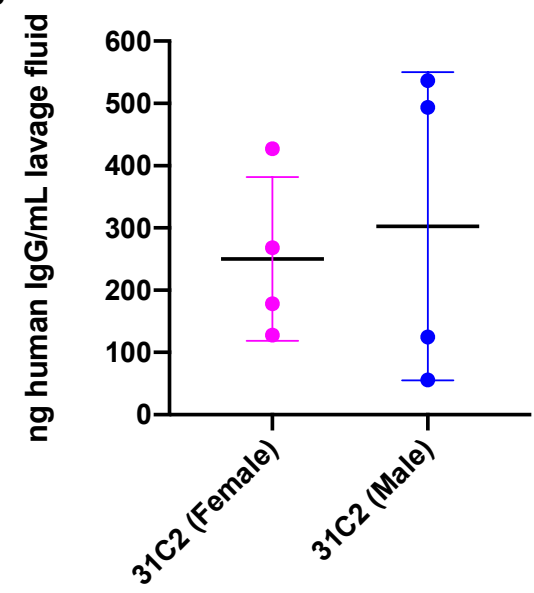

Figure 1. Evaluation of vectorized 31C2 monoclonal antibody. (A) 6-week-old male and female BALB/c mice $(n=4)$ were administered $2 \times 10^{10} \mathrm{VG}$ of AAV-mAb and plasma was monitored for hIgG expression over a period of 14 weeks. (B) BALF was collected at endpoint and hIgG was quantified. Data are represented as the mean \pm standard deviation.

\subsection{Safety and Tolerability of AAV6.2FF-31C2 in a Murine Model}

A mouse study was designed to investigate the safety and tolerability of the AAV6.2FF$31 \mathrm{C} 2$ vector administered to the muscle. The study design utilized three cohorts to evaluate endpoints at 7-, 28- and 56-days post AAV administration at a low $\left(1 \times 10^{11} \mathrm{VG}\right)$, mid $\left(2 \times 10^{11} \mathrm{VG}\right)$ and high $\left(6 \times 10^{11} \mathrm{VG}\right)$ doses $(n=6 ; 3$ males and 3 females) (Table 1$)$. Mice were dosed based on a fixed vector genome concentration; however, when adjusted for the weight the average dose per kilogram $(\mathrm{kg})$ was an average on $4.7 \times 10^{12}, 9.4 \times 10^{12}$ and $2.9 \times 10^{13} \mathrm{VG} / \mathrm{kg}$ for the low, mid and high dose groups, respectively (Figure S1). Due to the limited amount of blood that can be taken at one time point, only terminal blood draws were taken from the mice at endpoint for analysis.

Table 1. Mouse safety and tolerability study design.

\begin{tabular}{cccccc}
\hline Group & Cohort & Treatment & Dose (VG) & Endpoint & Number of Animals $^{\mathbf{2}}$ \\
\hline 1 & Day 7-low & AAV6.2FF-31C2 & $1 \times 10^{11}$ & Day 7 & $n=6$ \\
2 & Day 7-mid & AAV6.2FF-31C2 & $2 \times 10^{11}$ & Day 7 & $n=6$ \\
3 & Day 7-high & AAV6.2FF-31C2 & $6 \times 10^{11}$ & Day 7 & $n=6$ \\
4 & Day 7-mock & Vehicle only ${ }^{1}$ & - & Day 7 & $n=6$ \\
5 & Day 28-low & AAV6.2FF-31C2 & $1 \times 10^{11}$ & Day 28 & $n=6$ \\
6 & Day 28-mid & AAV6.2FF-31C2 & $2 \times 10^{11}$ & Day 28 & $n=6$ \\
7 & Day 28-high & AAV6.2FF-31C2 & $6 \times 10^{11}$ & Day 28 & $n=6$ \\
8 & Day 28-mock & Vehicle only ${ }^{1}$ & - & Day 28 & $n=6$ \\
9 & Day 56-low & AAV6.2FF-31C2 & $1 \times 10^{11}$ & Day 56 & $n=6$ \\
10 & Day 56-mid & AAV6.2FF-31C2 & $2 \times 10^{11}$ & Day 56 & $n=6$ \\
11 & Day 56-high & AAV6.2FF-31C2 & $6 \times 10^{11}$ & Day 56 & $n=6$ \\
12 & Day 56-mock & Vehicle only ${ }^{1}$ & - & Day 56 & $n=6$ \\
\hline
\end{tabular}

${ }^{1} 1 \times$ PBS-phosphate buffered saline was used as the vehicle, ${ }^{2} 3$ male and 3 female mice per group, - Not applicable. 
A dose dependent increase in hIgG transgene in murine plasma was observed between the low, mid and high dose cohorts across the three time points (days 7, 28 and 56) (Figure 2A). hIgG expression was highest 56 days post vector delivery resulting in average plasma hIgG concentrations of $67.9,127.7$ and $137.8 \mu \mathrm{g} / \mathrm{mL}$ for groups administered with low, mid and high doses, respectively. Interestingly, a minimal increase in plasma 31C2 was observed between groups administered with mid-dose and high-dose, despite a 3-fold increase in VG dose administered; however, it is possible that the high dose of AAV saturated the number of muscle cells in the leg susceptible to transduction. hIgG expression did not significantly alter murine $\operatorname{IgG}(\mathrm{mIgG})$ concentrations compared to vehicle treated control groups (Figure 2B). The average mIgG across day 7, 28 and 56 cohorts were 2.2, 3.3 and $2.3 \mathrm{mg} / \mathrm{mL}$, respectively (Figure $2 \mathrm{~B}$ ). The proportion of $\mathrm{hIgG}$ to $\mathrm{mIgG}$ increased from an average of $0.28-1.20 \%$ in the day 7 cohort to $0.72-4.25 \%$ in the day 28 cohort to $3.52-5.49 \%$ in the day 56 cohort (Figure 2B). All mice had a maximum of $9 \%$ of hIgG present except for two outliers in groups 9 (Day 56-low) and 11 (Day 56-high), each with high hIgG concentrations 212.4-251.9 $\mu \mathrm{g} / \mathrm{mL}$ and low mIgG concentrations 1.1-1.5 mg/mL, resulting in high hIgG proportions of $14.1 \%$ and $24 \%$ of $\mathrm{mIgG}$. All groups gained weight similarly to the vehicle controls throughout the course of the study, indicating general health was not negatively impacted by AAV6.2FF transfection and AAV6.2FF-mediated $\mathrm{mAb}$ expression (Figure 2D). Mice were terminally bled at endpoint and samples were submitted for leukocyte differential, clinical biochemistry and an inflammatory cytokine panel. Plasma alanine aminotransferase, aspartate aminotransferase, creatine kinase and creatinine concentrations were not significantly elevated across treatment groups for any cohort, indicating that liver and muscle damage was not present (Figure 2E-H). Serum albumin, globulin, total protein and urea concentrations were also evaluated (Figure S2).

Lymphocyte differential demonstrated no significant trends in total white blood cells, lymphocytes, neutrophils, monocytes or eosinophils between groups or cohorts, indicating a lack of lymphocyte based immune response captured within the time points of this study (Figure 2I,J and Figure S3). IFN $\gamma$ and TNF $\alpha$ were both elevated in all treatment groups, including mock groups, at day 7 , but the response subsided by day 28 , demonstrating that this response was acute and resolved quickly (Figure 2K,L). IL-1B, IL-2, IL-4, IL-6, IL-10, IL12p70, MCP-1 and GM-CSF concentrations were also evaluated but the trend for IFN $\gamma$ and $\mathrm{TNF} \alpha$ was not observed for other cytokines (Figure S4). The day seven increase was only significant for TNF $\alpha$ and none of the observed concentrations for this panel of cytokines are indicative of a strong inflammatory response. Nevertheless, these data demonstrate there was a slight elevation in select cytokines that resolved by day 28. Overall, this mouse study revealed no significant changes in blood chemistry or hematological parameters as a result of vectorized $31 \mathrm{C} 2$ expression.

Tissues from the high dose groups, euthanized 56 days following AAV-31C2 injection, were used for biodistribution analysis. Unfortunately, due to the small size of mouse gastrocnemius muscles, the entire sample was submitted for histology and was therefore not included here. While AAV vector genomes were detected in all tissues examined, only in the kidney and liver was there a significant, dose dependent increase in AAV genome copy numbers (Figure S5). 
A

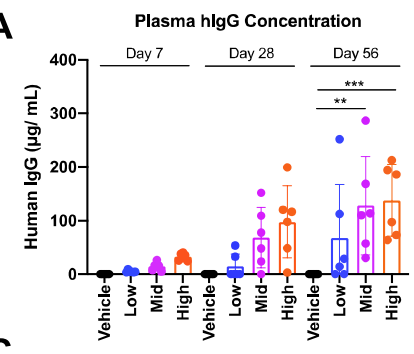

C

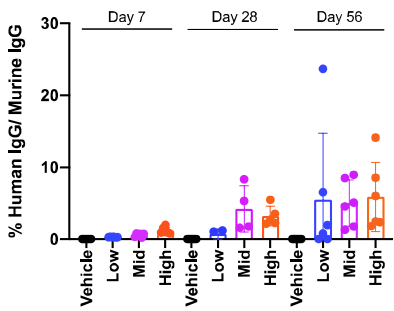

E

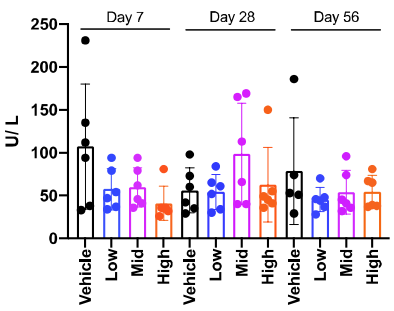

G

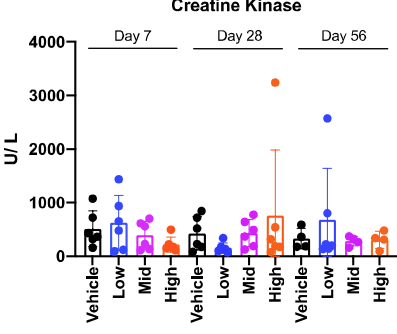

I

White Blood Cells

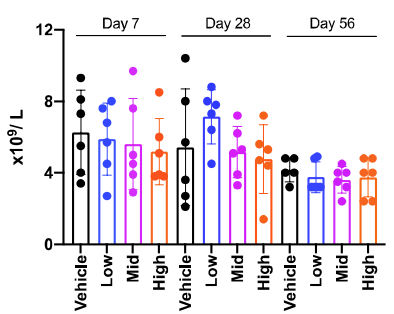

K

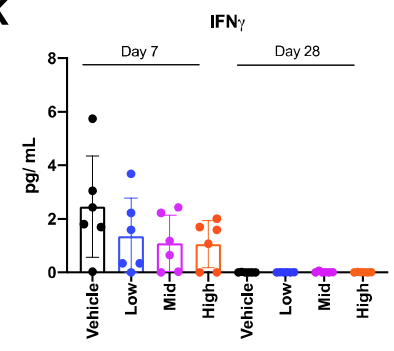

B
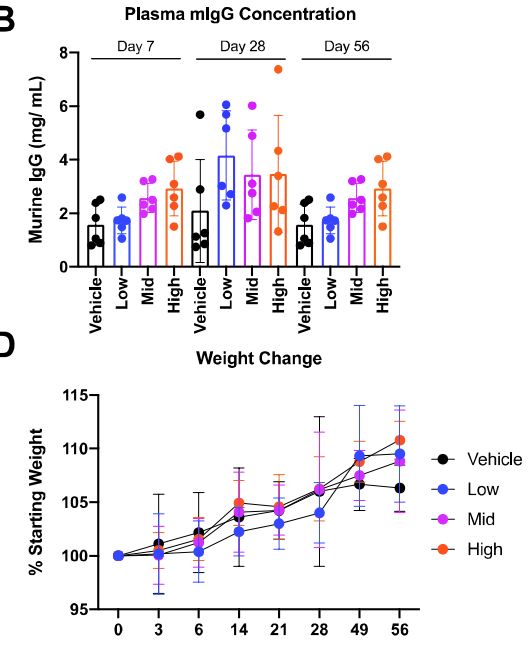

F

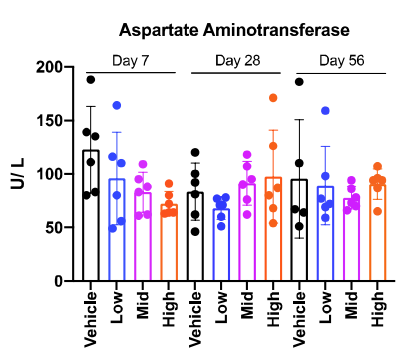

H

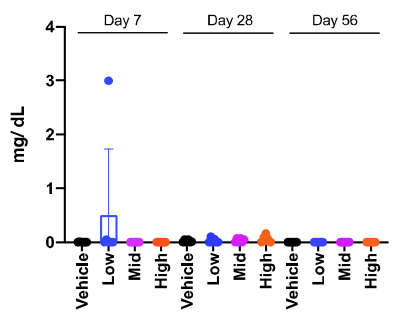

J

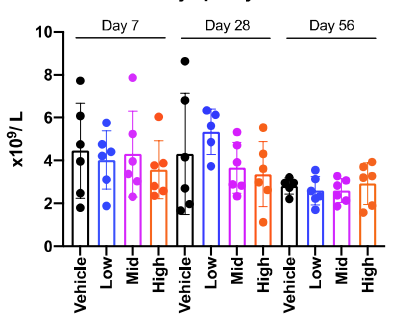

L

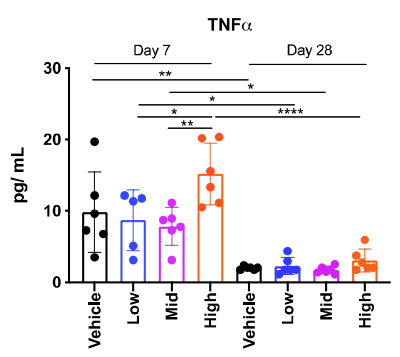

Figure 2. Findings of the murine AAV6.2FF-31C2 safety and tolerability study. 6-week-old male and female BALB/c mice ( $n=6$; equal male and female mice per group) were administered either a low $\left(1 \times 10^{11} \mathrm{VG}\right)$, mid $\left(2 \times 10^{11} \mathrm{VG}\right)$ or high $\left(6 \times 10^{11} \mathrm{VG}\right)$ dose and sacrificed either 7-, 28 - or 56 -days 
post AAV administration. Terminal blood samples were collected at endpoint and plasma was analyzed for (A) hIgG concentration, (B) $\mathrm{mIgG}$ concentration and (C) the ratio of hIgG to $\mathrm{mIgG}$ is shown. (D) Mice were weighted on a weekly/ biweekly schedule throughout the in-life phase of the study. Plasma and whole blood samples were analyzed for (E) alanine aminotransferase, (F) aspartate aminotransferase, $(\mathbf{G})$ creatine kinase, $(\mathbf{H})$ creatinine, $(\mathbf{I})$ white blood cells, (J) lymphocytes, IFN $\gamma$ (K) and TNF $\alpha(\mathbf{L})$. A one-way ANOVA was used for analysis. Data are represented as the mean \pm standard deviation. ${ }^{*} p<0.05,{ }^{* *} p<0.01,{ }^{* * *} p<0.001$ and ${ }^{* * * *} p<0.0001$.

\subsection{Safety and Tolerability of AAV6.2FF-31C2 in a Pediatric Ovine (Sheep) Model}

An additional goal of the sheep study was to evaluate the feasibility of the AAV$\mathrm{mAb}$ approach in a large, outbred animal model to support the data previously generated using this platform in rodent models. Three two-week-old male lambs were administered $5 \times 10^{12} \mathrm{VG} / \mathrm{kg}$ of AAV6.2FF-31C2 by IM injections to the rump. An additional three two-week-old male lambs served as controls. The lambs weighed $4.22,5.94$ and $7.12 \mathrm{~kg}$ at the time of vector administration and required two to three injections to administer the total volume of AAV vector. Lambs were evaluated for a four week in-life phase prior to endpoint to evaluate pathology. Blood was drawn on a weekly basis to evaluate transgene expression and plasma hIgG concentrations increased steadily throughout the study, peaking at 21.4, 33.7 and $46.7 \mu \mathrm{g} / \mathrm{mL}$ at the final time point (Figure 3A). Ovine $\operatorname{IgG}(\mathrm{oIgG})$ concentrations were also evaluated to determine the ratio of $\mathrm{hIgG}$ to oIgG (Figure 3B,C). The newborn lambs in the study received colostrum and were removed from the mother ewes after approximately 48 hours; at which point they were adapted to bottle milk supplemented with bovine dried colostrum. Therefore, the higher level of oIgG in Sheep 8486 and 8491 could be attributed to these animals having received more colostrum than the other sheep in this study.

The hIgG transgene represents much less than $0.1 \%$ of the total oIgG due to the larger blood volume compared to mice. Lambs gained weight as expected throughout the in-life phase on the study, more than doubling in weight over 28 days (Figure 3D). Interestingly, the level of hIgG increased despite this rapid weight increase, demonstrating efficient expression of the transgene in this sheep model.

In the lung tissue homogenates, Sheep 8484 and 8491 had higher levels of hIgG than 8486, reflecting the trend of hIgG levels observed in the plasma, similar to the pattern observed previously in murine lung lavages (Figure 3E). These results confirm that delivery of AAV6.2FF-31C2 to the muscle results in antibody diffusion to the lung, the primary organ involved in respiratory infections. Low levels of anti-AAV6.2FF capsid antibodies were detected in each of the sheep, which show an upward trend over the 28-day sampling period (Figure 3F).

Data was collected to assess safety and tolerability of this large animal model feasibility study. Plasma creatine kinase, alanine aminotransferase, and aspartate amino transferase did not demonstrate any notable increases above the reference ranges and in comparison, to sham control sheep (Figure 3G-J). Similarly, lactate dehydrogenase, urea, glucose, bilirubin, total protein, globulin and albumin and plasma alkaline phosphate concentrations remained steady or within the references ranges throughout the study and in comparison, to sham control sheep (Figure S6). White blood cell and lymphocyte counts trended slightly upwards but remained within the ovine reference range (Figure 3K,L). Red blood cell count, hemoglobin and hemocrit concentrations as well as platelets, neutrophil, monocyte, eosinophil and basophil counts were also monitored with few notable deviations from controls (Figure S7). In the AAV6.2FF-31C2 group, there was a spike in neutrophils in two out of three sheep on day seven that returned to baseline by day 14; however, the values were within the upper range of the reference interval.

To better understand the biodistribution of AAV6.2FF-31C2 following IM injections in tissues other than the vector injected muscle, tissues collected from sheep euthanized 28 days post AAV6.2FF-31C2 injection were analyzed for AAV vector genome biodistribu- 
tion (Figure S8). Only in the kidney, heart, brain, and spleen of sheep \# 8491 were AAV vector genomes above background levels.

A

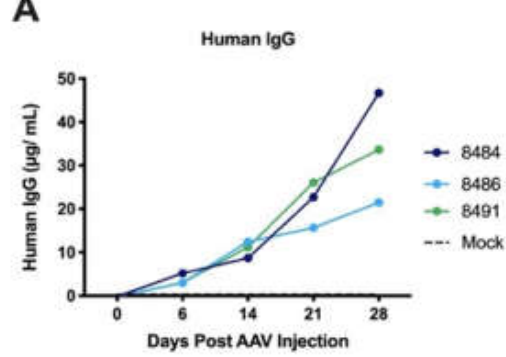

D

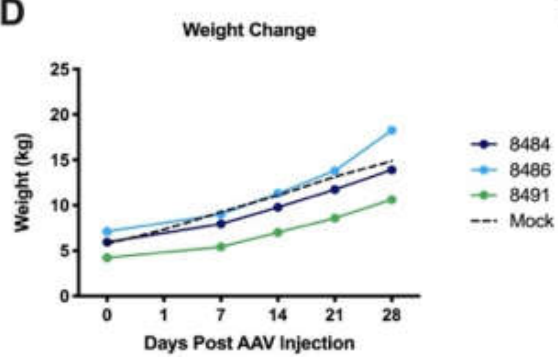

G

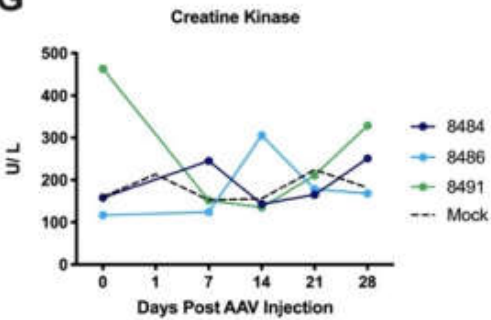

J

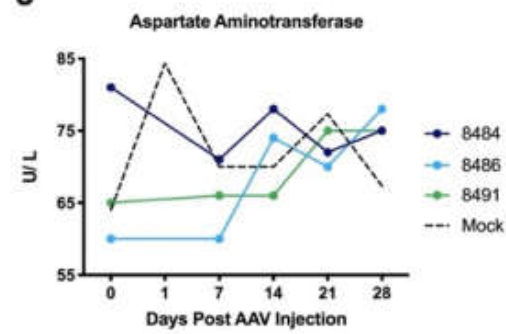

B

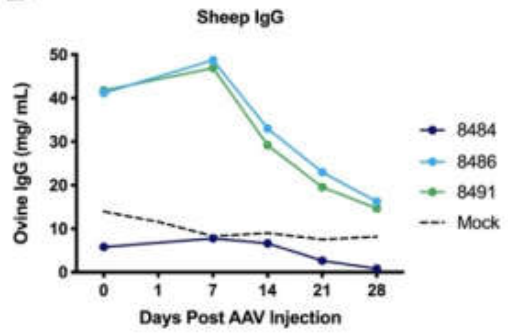

E

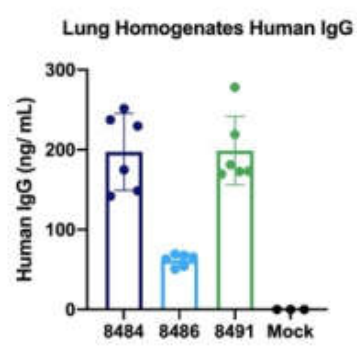

H
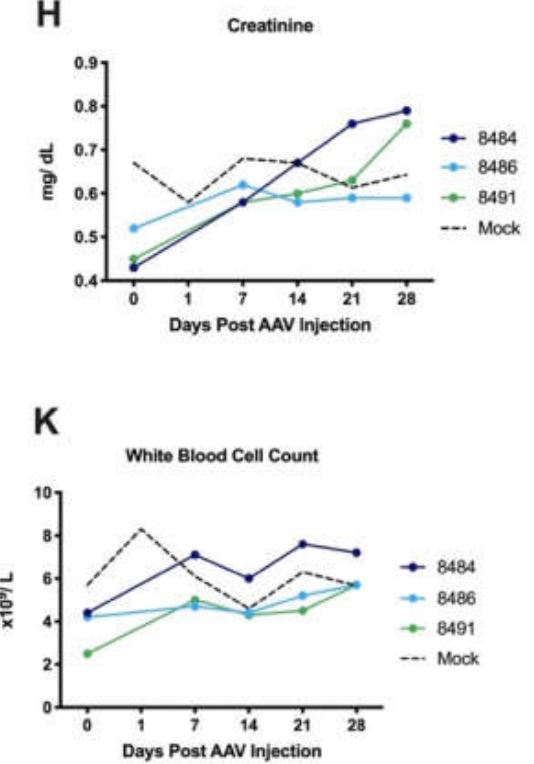

C

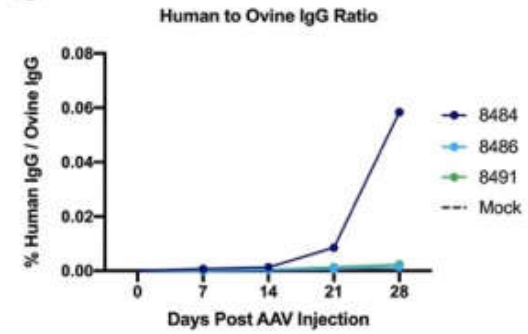

$\mathbf{F}$

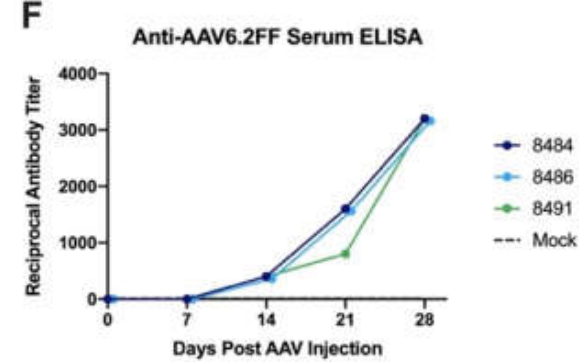

I

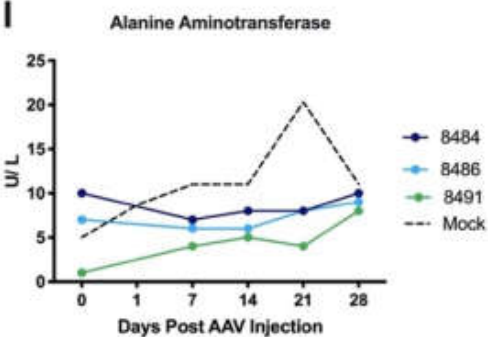

L

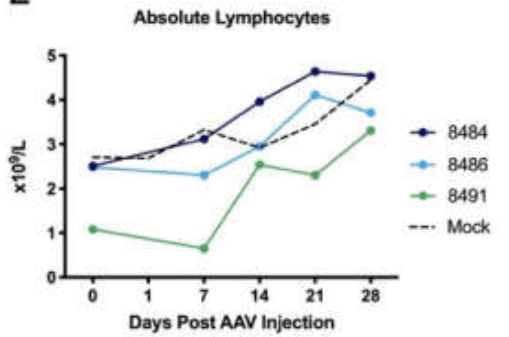

Figure 3. Findings of the ovine AAV6.2FF-31C2 feasibility study. Three 2-week-old lambs were administered $5 \times 10^{12} \mathrm{VG} / \mathrm{kg}$ of AAV6.2FF-31C2 by IM injections to the rump. Aged-matched controls $(n=3)$ were administered PBS IM. Weekly blood samples were collected and analyzed for plasma (A) hIgG concentration, (B) oIgG concentration and (C) the calculated ratio of hIgG to oIgG is displayed. (D) Sheep were weighted weekly throughout the in-life phase of the study. (E) Lung homogenates at endpoint (two sample sites per animal) were analyzed for hIgG concentration. (F) Anti-AAV6.2FF capsid antibodies were monitored weekly. Plasma and whole blood samples were analyzed for $(\mathbf{G})$ creatine kinase, $(\mathbf{H})$ creatinine, (I) alanine aminotransferase, (J) aspartate aminotransferase, (K) white blood cells and (L) absolute lymphocytes. The black dotted line represents the average value for the three control sheep.

\subsection{Lack of Pathology Observed in Murine and Ovine Models Following Intramuscular $A A V-m A b$ Administration}

We examined murine tissues histologically to determine whether AAV-31C2 led to any adverse effects in the lung, heart, brain, kidney, spleen, liver or gastrocnemius 
muscle. Mild inflammation of the muscle at the site of injection was observed in all groups, with the exception of the vehicle only groups, indicating some inflammation was induced by the vector rather than from the injection alone. Only four mice exhibited inflammation extending to $>50 \%$ of the muscle tissue, including mice from low and high dose groups (Figure 4A,B) (Supplemental Table S1). These findings were attributed to the IM route of AAV injections. All liver tissue sections showed mild lipid-type vacuolations mainly in centrilobular spaces, not indicative of pathological origin. The brain and kidney demonstrated normal histology, with no indication of toxicity. Minor irregularities of other organs included mild mineralization and scattered lymphocytes in the epicardium, and extramedullary hematopoiesis (EMH) in the lung. No evidence of lesions or other pathological changes was observed in these organs; therefore, these observations were deemed incidental findings.

Tissues from sheep administered AAV6.2FF-31C2 were similarly examined to determine if any toxicity was attributed to the vector. Mild inflammation of striated muscle tissue was observed in the node nerve, intestines, lymph node, thymus, skin and muscle. Only muscle tissue found directly at the site of injection and in intestine tissue of Sheep 8486 was considered greatly affected, although all striated muscle was deemed clinically insignificant (Figure 4C). These findings are consistent with the lack of gross lesions and other clinical observations.

Sheep 8484 showed mild inflammation of the epicardium, while all animals showed EMH in the liver and the presence of mild interstitial lymphocytes in the kidney. These findings were considered incidental and can be attributed to hematopoiesis and lymphopoiesis seen in lambs. None of the tissues sampled from the large intestine, small intestine, abomasum, lymph node, brain, spleen, lung, testis, bladder, pancreas, thymus, liver, kidney, bone marrow, thyroid, parathyroid, heart, skin, and muscle tissue showed any signs of vector-related toxicities. Overall, none of the organs observed in both the murine and ovine models showed any signs of adverse clinical effects linked to AAV6.2FF-31C2 treatment.
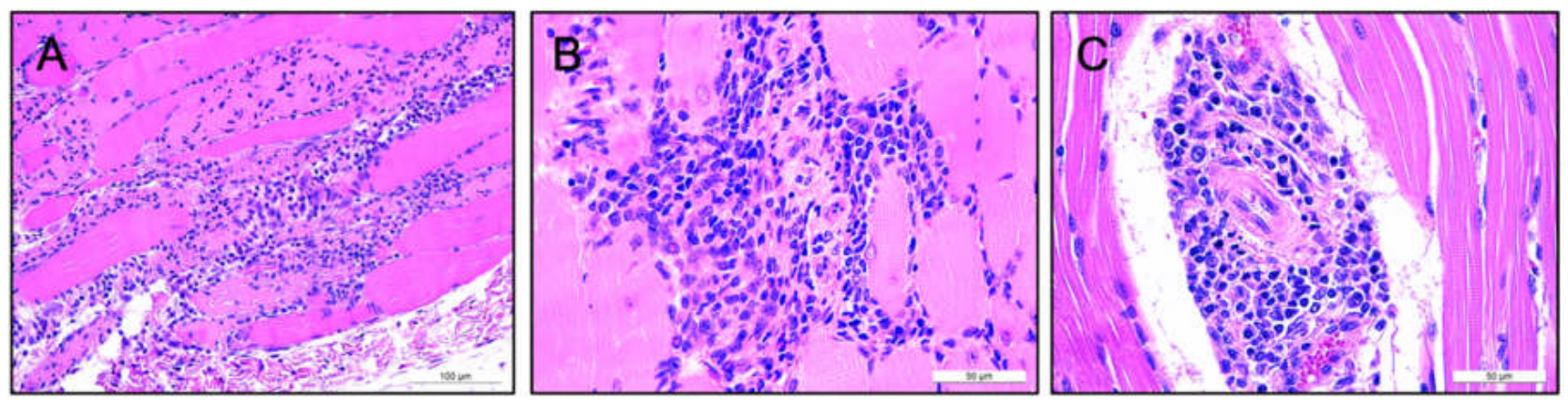

Figure 4. Comparison of striated murine and ovine muscle tissue using H\&E staining. Histopathological images of inflamed murine muscle tissue 28 days post IM administered of $5 \times 10^{12} \mathrm{VG} / \mathrm{kg}$ of AAV-31C2 at $20 \times($ A) and $40 \times($ B), showing lymphocytes and macrophages in the endomysium surrounding numerous myofibers. Straited muscle tissue from Sheep \#8486 28 days post IM administered of $5 \times 10^{12} \mathrm{VG} / \mathrm{kg}$ of AAV-31C2 is seen at $40 \times(\mathrm{C})$. Scattered lymphocytes, plasma cells and rare macrophages were present surrounding myofibers, with accumulation seen in the endomysium and medium-size vessels.

\subsection{Human IgG Is Expressed in Murine and Ovine Muscle}

While quantification of hIgG in the plasma demonstrates successful vectorized antibody expression, we sought to confirm expression at the site of injection. Muscle samples from murine and ovine subjects were prepared for histology and immunohistochemical staining of hIgG was conducted. Widespread hIgG staining was observed in the skeletal muscle surrounding the site of IM AAV6.2FF-31C2 administration for both species (Figure 5). 

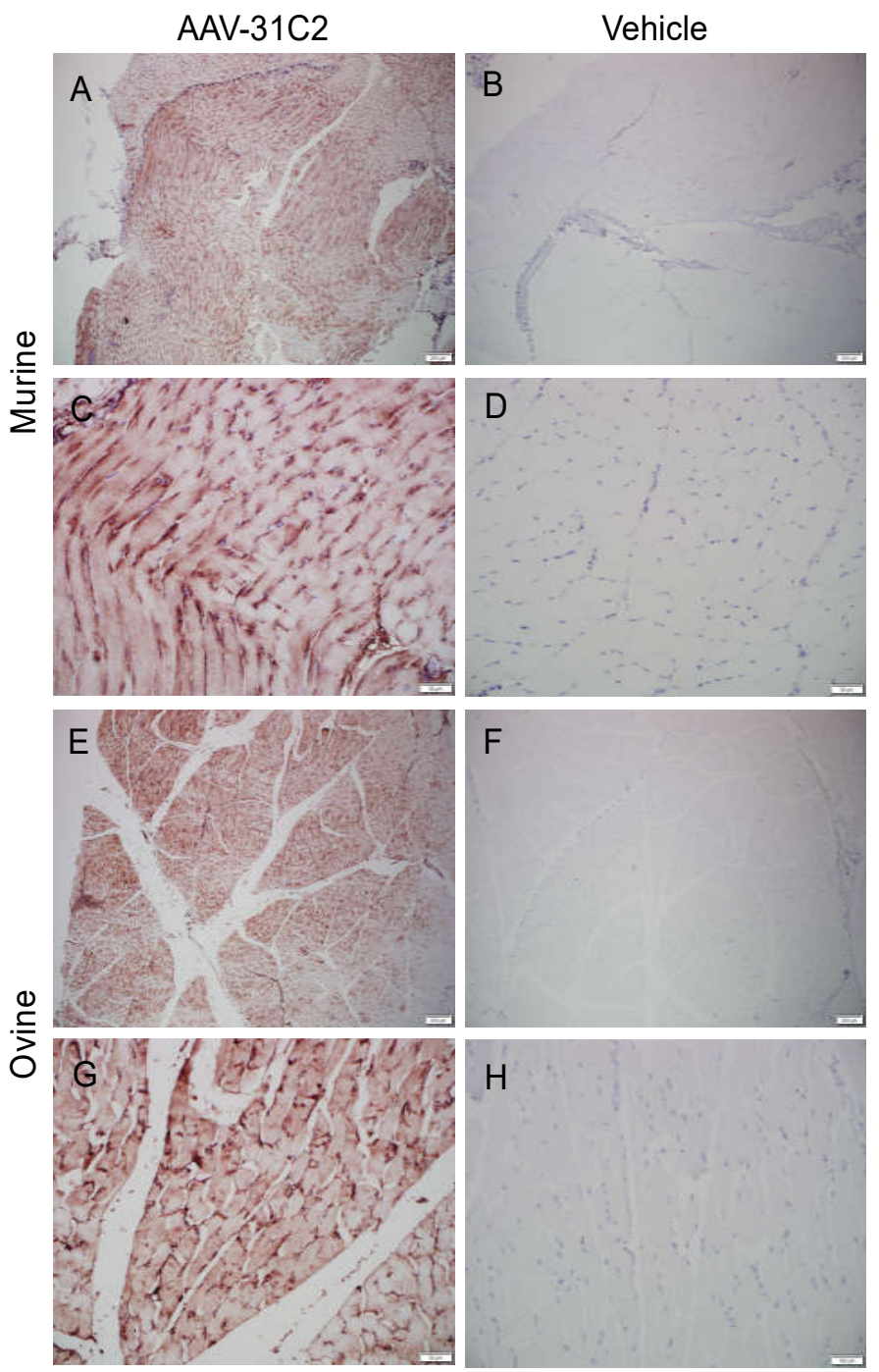

Figure 5. Immunohistochemical staining of human $\operatorname{IgG}$ in murine and ovine muscle. Stained murine muscle tissue from a mouse intramuscularly injected with $5 \times 10^{12} \mathrm{VG} / \mathrm{kg}$ AAV6.2FF-31C2 28 days prior at (A) $4 \times$ and (C) $20 \times$ and negative control murine muscle receiving vehicle only at (B) $4 \times$ and (D) $20 \times$ Stained ovine muscle tissue from sheep \#8486 28 days post injection with $5 \times 10^{12} \mathrm{VG} / \mathrm{kg}$ AAV6.2FF-31C2 at (E) $4 \times$ and (G) $20 \times$ and healthy negative control tissue at (F) $4 \times$ and (H) $20 \times$.

\section{Discussion}

Vectorized long-term expression of therapeutic antibodies provides a promising alternative to traditional immunizations. Classical antigen-based vaccines require a robust endogenous immune response to provide immunity and the age-related decline in the immune function in those age 65 or older, as well as immunocompromised individuals, leads to increased vulnerability to infection and a decrease in vaccine responsiveness [20,21]. Studies show the effectiveness of influenza vaccines are reduced as much as 50\% in those 65 years of age or older compared to young adults [22-24]. Here we describe safety, tolerability and feasibility of utilizing the AAV6.2FF capsid to express SARS-CoV-2 mAb 31C2 in both murine and ovine animal models.

All cohorts of mice displayed tolerability to increasing dosages of AAV6.2FF-31C2, with no notable pathology. Any alterations in cytokine levels in the mouse tox study were resolved after the day 7 time point; however, it is probable that the peak of the cytokine response occurred well before the first cohort endpoint. Regarding future studies, cytokine responses at early time points (e.g., 2 to 12 hours post-AAV administration) would shed light on the extent to which IM administration of AAV6.2FF activates the innate immune 
response. Elevated levels of IFN $\gamma$ and $\mathrm{TNF} \alpha$ were detected in the vehicle only treated groups of mice as well as in the mice receiving AAV, suggesting that the injection itself might have induced this response. Analysis of the biodistribution of AAV in murine tissues revealed a statistically significant dose-dependent increase in AAV vector genomes in the liver and kidney, which is not surprising as both are organs with high blood flow. However, higher levels of AAV genomes in the liver and kidney did not have an impact on the tolerability of AAV.

Previous non-human primate (NHP) studies have shown that AAV expression of monoclonal antibodies against simian immunodeficiency virus (SIV) is an effective method for prolonged antibody-based immunity to challenge $[25,26]$. Stable vectorized expression of the anti-SIV monoclonal antibody 5L7 has been maintained in NHPs at 240-350 $\mu \mathrm{g} / \mathrm{mL}$ for over 6 years [25]. While our study was terminated after 4 weeks to evaluate pathology, long term expression studies in the sheep model are currently ongoing. AAV8-mediated delivery of two anti-SIV mAbs directed against the Env protein resulted in peak plasma concentrations of 21.2 and $8.6 \mu \mathrm{g} / \mathrm{mL}$ while providing protection from weekly limiting dose intrarectal SIV challenges [27]. In our sheep study, we observed a steady weekly increase in 31C2, which peaked as high as $46.7 \mu \mathrm{g} / \mathrm{mL}$ after 28 days, despite the rapid > 2.3-fold weight gain post vector administration. Although NHPs are an ideal large animal model due to their similarity to humans, adult macaques generally weigh less than $20 \mathrm{~kg}$, a mass lambs reached in a matter of weeks with adult sheep weighing more than $80 \mathrm{~kg}$, which is a more clinically relevant model in terms of human size, blood volume and lung physiology. Continued monitoring of the sheep to determine longevity of AAV-mediated expression of 31C2 in the plasma as the lambs grew to full-sized adults would provide insight into how well AAV6.2FF could express mAbs in an adult human.

One of the limitations of this study was the fact that we were unable to perform bronchiolar lavages on the sheep. While lung tissue from the sheep was retrieved and analyzed for hIgG expression, quantification of hIgG in BALF would be very informative and would have allowed us to compare the proportion of hIgG in serum vs. lung fluid, like we did in the mice.

To the best of our knowledge, this is the first published data regarding the safety and tolerability of AAV-mediated antibody expression in vivo. However, there are numerous reports evaluating AAV-based therapies for other applications, demonstrating the strong safety profile of AAV vectors [28-30]. In mice, mild and acute liver pathology has been observed following systemic delivery of AAV8 and AAV9 gene therapy vectors at $6 \times 10^{13}$ and $1 \times 10^{14} \mathrm{VG} / \mathrm{kg}$; however, these were toxicology studies designed to understand the potential toxicities associated with high clinical doses while this study focused on vector tolerability at a functional dose [31,32].

Overall, our results exhibited a lack of significant alterations in serum biochemistry or histopathological findings in both murine and ovine studies, suggesting AAV vectorized antibody expression is a safe approach to generate immunity. Given the absence of notable toxicity findings in combination with the demonstration of large animal model feasibility, further development of the AAV6.2FF-mAb platform is warranted, including the evaluation of anti-drug antibody (ADA) responses and efficacy studies in non-human primate challenge models.

Supplementary Materials: The following are available online at https:/ / www.mdpi.com/article / 10.3390/biomedicines9091186/s1, Figure S1: Calculated murine AAV6.2FF-31C2 vector genome dose by weight, Figure S2: Additional murine clinical chemistry, Figure S3: Additional murine hematology, Figure S4: Additional murine cytokine profiles, Figure S5: Biodistribution of AAV in Murine Tissues following IM administration of AAV6.2FF-31C2, Figure S6: Additional ovine clinical chemistry, Figure S7: Additional ovine hematology, Figure S8: Biodistribution of AAV in Ovine Tissues following IM administration of AAV6.2FF-31C2, Table S1: Mouse H\&E Grading, Table S2: Sheep H\&E Grading. 
Author Contributions: Conceptualization, A.D.R., L.P.v.L., B.T. and S.K.W.; methodology, A.D.R., L.P.v.L. and B.M.M. produced the vector and executed the study designs., J.A.L., N.Z., E.M.B., S.P.T., B.A.Y.S., J.G.E.Y., Y.P., B.W.B. and L.S. assisted with animal work, vector production and/or data analysis. H.F.-B. and G.P.K. generated antibody 31C2 and provided the antibody sequence for this monoclonal antibody. L.S. performed the pathology analysis.; writing-original draft preparation, A.D.R. and L.P.v.L.; writing-review and editing, B.M.W., D.K., K.K., B.W.B., L.S., and S.K.W.; supervision, S.K.W.; funding acquisition, S.K.W. All authors have read and agreed to the published version of the manuscript.

Funding: Funding for this study was provided by Avamab Pharma Inc. A.D.R. was the recipient of a Mitacs Accelerate Studentship for her work in partnership with Avamab Pharma Inc.

Institutional Review Board Statement: This study was conducted according to the guidelines of the Canadian Council on Animal Care (CCAC), and approved by the Animal Care Committee of the University of Guelph (Animal Use Protocol numbers 3827 and 4186, approved 22 September 2017 and 1 April 2020, respectively).

Data Availability Statement: Data is contained within the article or supplementary material.

Acknowledgments: We would like to thank all those who were involved in the care of the animals for these studies.

Conflicts of Interest: LPvL and SKW are inventors on a US patent for the AAV6.2FF capsid. This patent (US20190216949) is licensed to Avamab Pharma Inc., where BT, LPvL and SKW are co-founders and BT serves as an executive. The funders had no role in the design of the study; in the collection, analyses, or interpretation of data; in the writing of the manuscript, or in the decision to publish the results.

\section{References}

1. Gavor, E.; Choong, Y.K.; Er, S.Y.; Sivaraman, H.; Sivaraman, J. Structural Basis of SARS-CoV-2 and SARS-CoV Antibody Interactions. Trends Immunol. 2020, 41, 1006-1022. [CrossRef] [PubMed]

2. Olchanski, N.; Hansen, R.N.; Pope, E.; D’Cruz, B.; Fergie, J.; Goldstein, M.; Krilov, L.R.; McLaurin, K.K.; Nabrit-Stephens, B.; Oster, G.; et al. Palivizumab Prophylaxis for Respiratory Syncytial Virus: Examining the Evidence Around Value. Open Forum Infect. Dis. 2018, 5, ofy031. [CrossRef] [PubMed]

3. Lu, R.-M.; Hwang, Y.-C.; Liu, I.-J.; Lee, C.-C.; Tsai, H.-Z.; Li, H.-J.; Wu, H.-C. Development of therapeutic antibodies for the treatment of diseases. J. Biomed. Sci. 2020, 27, 1-30. [CrossRef]

4. Zost, S.J.; Gilchuk, P.; Case, J.B.; Binshtein, E.; Chen, R.E.; Nkolola, J.P.; Schäfer, A.; Reidy, J.X.; Trivette, A.; Nargi, R.S.; et al. Potently neutralizing and protective human antibodies against SARS-CoV-2. Nat. Cell Biol. 2020, 584, 443-449. [CrossRef] [PubMed]

5. Zost, S.J.; Gilchuk, P.; Chen, R.E.; Case, J.B.; Reidy, J.X.; Trivette, A.; Nargi, R.S.; Sutton, R.E.; Suryadevara, N.; Chen, E.C.; et al. Rapid isolation and profiling of a diverse panel of human monoclonal antibodies targeting the SARS-CoV-2 spike protein. Nat. Med. 2020, 26, 1422-1427. [CrossRef] [PubMed]

6. Wang, C.; Li, W.; Drabek, D.; Okba, N.M.A.; van Haperen, R.; Osterhaus, A.D.M.E.; van Kuppeveld, F.J.M.; Haagmans, B.L.; Grosveld, F.; Bosch, B.-J. A human monoclonal antibody blocking SARS-CoV-2 infection. Nat. Commun. 2020, 11, 1-6. [CrossRef] [PubMed]

7. Chen, P.; Nirula, A.; Heller, B.; Gottlieb, R.L.; Boscia, J.; Morris, J.; Huhn, G.; Cardona, J.; Mocherla, B.; Stosor, V.; et al. SARS-CoV-2 Neutralizing Antibody LY-CoV555 in Outpatients with Covid-19. N. Engl. J. Med. 2021, 384, 229-237. [CrossRef] [PubMed]

8. Yang, L.; Wang, P. Passive Immunization against HIV/AIDS by Antibody Gene Transfer. Viruses 2014, 6, 428-447. [CrossRef] [PubMed]

9. Rghei, A.D.; Van Lieshout, L.P.; Santry, L.A.; Guilleman, M.M.; Thomas, S.P.; Susta, L.; Karimi, K.; Bridle, B.W.; Wootton, S.K. AAV Vectored Immunoprophylaxis for Filovirus Infections. Trop. Med. Infect. Dis. 2020, 5, 169. [CrossRef]

10. Lewis, A.D.; Chen, R.; Montefiori, D.C.; Johnson, P.R.; Clark, K.R. Generation of Neutralizing Activity against Human Immunodeficiency Virus Type 1 in Serum by Antibody Gene Transfer. J. Virol. 2002, 76, 8769-8775. [CrossRef]

11. Martinez-Navio, J.M.; Fuchs, S.P.; Pantry, S.N.; Lauer, W.A.; Duggan, N.N.; Keele, B.F.; Rakasz, E.G.; Gao, G.; Lifson, J.D.; Desrosiers, R.C. Adeno-Associated Virus Delivery of Anti-HIV Monoclonal Antibodies Can Drive Long-Term Virologic Suppression. Immun. 2019, 50, 567-575.e5. [CrossRef]

12. Limberis, M.P.; Adam, V.S.; Wong, G.; Gren, J.; Kobasa, D.; Ross, T.M.; Kobinger, G.P.; Tretiakova, A.; Wilson, J.M. Intranasal Antibody Gene Transfer in Mice and Ferrets Elicits Broad Protection Against Pandemic Influenza. Sci. Transl. Med. 2013, 5, 187ra72. [CrossRef] 
13. Van Lieshout, L.P.; Soule, G.; Sorensen, D.; Frost, K.L.; He, S.; Tierney, K.; Safronetz, D.; A Booth, S.; Kobinger, G.P.; Qiu, X.; et al. Intramuscular Adeno-Associated Virus-Mediated Expression of Monoclonal Antibodies Provides 100\% Protection Against Ebola Virus Infection in Mice. J. Infect. Dis. 2018, 217, 916-925. [CrossRef]

14. Guilleman, M.M.; Stevens, B.A.Y.; Van Lieshout, L.P.; Rghei, A.D.; Pei, Y.; Santry, L.A.; Thompson, B.; Wootton, S.K. AAV-mediated delivery of actoxumab and bezlotoxumab results in serum and mucosal antibody concentrations that provide protection from $\mathrm{C}$. difficile toxin challenge. Gene Ther. 2021, 1-8. [CrossRef]

15. Miesbach, W.; Meijer, K.; Coppens, M.; Kampmann, P.; Klamroth, R.; Schutgens, R.; Tangelder, M.; Castaman, G.; Schwäble, J.; Bonig, H.; et al. Gene therapy with adeno-associated virus vector 5-human factor IX in adults with hemophilia B. Blood 2018, 131, 1022-1031. [CrossRef]

16. Penaud-Budloo, M.; François, A.; Clément, N.; Ayuso, E. Pharmacology of Recombinant Adeno-associated Virus Production. Mol. Ther. Methods Clin. Dev. 2018, 8, 166-180. [CrossRef] [PubMed]

17. van Lieshout, L.P.; Domm, J.M.; Rindler, T.N.; Frost, K.L.; Sorensen, D.L.; Medina, S.J.; Booth, S.A.; Bridges, J.; Wootton, S.K. A Novel Triple-Mutant AAV6 Capsid Induces Rapid and Potent Transgene Expression in the Muscle and Respiratory Tract of Mice. Mol. Ther. Methods Clin. Dev. 2018, 9, 323-329. [CrossRef] [PubMed]

18. Balazs, A.; Chen, J.; Hong, C.M.; Rao, D.; Yang, L.; Baltimore, D. Antibody-based protection against HIV infection by vectored immunoprophylaxis. Nat. Cell Biol. 2011, 481, 81-84. [CrossRef]

19. Halbert, C.L.; Allen, J.M.; Miller, A.D. Efficient mouse airway transduction following recombination between AAV vectors carrying parts of a larger gene. Nat. Biotechnol. 2002, 20, 697-701. [CrossRef]

20. Gustafson, C.; Kim, C.; Weyand, C.M.; Goronzy, J.J. Influence of immune aging on vaccine responses. J. Allergy Clin. Immunol. 2020, 145, 1309-1321. [CrossRef]

21. Aiello, A.; Farzaneh, F.; Candore, G.; Caruso, C.; Davinelli, S.; Gambino, C.M.; Ligotti, M.E.; Zareian, N.; Accardi, G. Immunosenescence and Its Hallmarks: How to Oppose Aging Strategically? A Review of Potential Options for Therapeutic Intervention. Front. Immunol. 2019, 10, 2247. [CrossRef]

22. Jefferson, T.; Di Pietrantonj, C.; Al-Ansary, L.; Ferroni, E.; Thorning, S.; E Thomas, R. Vaccines for preventing influenza in the elderly. Cochrane Database Syst. Rev. 2010, CD004876. [CrossRef]

23. Michiels, B.; Govaerts, F.; Remmen, R.; Vermeire, E.; Coenen, S. A systematic review of the evidence on the effectiveness and risks of inactivated influenza vaccines in different target groups. Vaccine 2011, 29, 9159-9170. [CrossRef]

24. Demicheli, V.; Jefferson, T.; Di Pietrantonj, C.; Ferroni, E.; Thorning, S.; E Thomas, R.; Rivetti, A. Vaccines for preventing influenza in the elderly. Cochrane Database Syst. Rev. 2018, 2, CD004876. [CrossRef] [PubMed]

25. Martinez-Navio, J.M.; Fuchs, S.P.; Mendes, D.E.; Rakasz, E.G.; Gao, G.; Lifson, J.D.; Desrosiers, R.C. Long-Term Delivery of an Anti-SIV Monoclonal Antibody With AAV. Front. Immunol. 2020, 11, 449. [CrossRef] [PubMed]

26. Fuchs, S.P.; Martinez-Navio, J.M.; Piatak, M., Jr.; Lifson, J.D.; Gao, G.; Desrosiers, R.C. AAV-Delivered Antibody Mediates Significant Protective Effects against SIVmac239 Challenge in the Absence of Neutralizing Activity. PLOS Pathog. 2015, 11, e1005090. [CrossRef]

27. Welles, H.C.; Jennewein, M.F.; Mason, R.D.; Narpala, S.; Wang, L.; Cheng, C.; Zhang, Y.; Todd, J.-P.; Lifson, J.D.; Balazs, A.B.; et al. Vectored delivery of anti-SIV envelope targeting mAb via AAV8 protects rhesus macaques from repeated limiting dose intrarectal swarm SIVsmE660 challenge. PLOS Pathog. 2018, 14, e1007395. [CrossRef] [PubMed]

28. Nathwani, A.C.; Reiss, U.M.; Tuddenham, E.G.; Rosales, C.; Chowdary, P.; McIntosh, J.; Della Peruta, M.; Lheriteau, E.; Patel, N.; Raj, D.; et al. Long-Term Safety and Efficacy of Factor IX Gene Therapy in Hemophilia B. N. Engl. J. Med. 2014, 371, 1994-2004. [CrossRef] [PubMed]

29. Russell, S.; Bennett, J.; A Wellman, J.; Chung, D.C.; Yu, Z.-F.; Tillman, A.; Wittes, J.; Pappas, J.; Elci, O.; McCague, S.; et al. Efficacy and safety of voretigene neparvovec (AAV2-hRPE65v2) in patients with RPE65 -mediated inherited retinal dystrophy: A randomised, controlled, open-label, phase 3 trial. Lancet 2017, 390, 849-860. [CrossRef]

30. Jacobson, S.G. Gene Therapy for Leber Congenital Amaurosis Caused by RPE65 Mutations. Arch. Ophthalmol. 2012, 130, 9-24. [CrossRef]

31. Meadows, A.S.; Duncan, F.J.; Camboni, M.; Waligura, K.; Montgomery, C.; Zaraspe, K.; Naughton, B.J.; Bremer, W.G.; Shilling, C.; Walker, C.M.; et al. A GLP-Compliant Toxicology and Biodistribution Study: Systemic Delivery of an rAAV9 Vector for the Treatment of Mucopolysaccharidosis IIIB. Hum. Gene Ther. Clin. Dev. 2015, 26, 228-242. [CrossRef] [PubMed]

32. Greig, J.A.; Limberis, M.P.; Bell, P.; Chen, S.-J.; Calcedo, R.; Rader, D.J.; Wilson, J.M. Nonclinical Pharmacology/Toxicology Study of AAV8.TBG.mLDLR and AAV8.TBG.hLDLR in a Mouse Model of Homozygous Familial Hypercholesterolemia. Hum. Gene Ther. Clin. Dev. 2017, 28, 28-38. [CrossRef] [PubMed] 